\title{
Monitoring hormone replacement therapy by biochemical markers of bone metabolism in menopausal women
}

\section{E Dogan, C Posaci}

Biochemical markers of bone metabolism are divided into two groups: formation and resorption markers. Bone turnover is a dynamic process, which increases in postmenopausal period. Hormone replacement therapy (HRT) can diminish this increased bone turnover. Biochemical markers reflect acute changes in bone metabolism. Therefore, they may be very useful for the prediction of subsequent bone mineral density changes after HRT in menopausal women. Both oral and transdermal routes of HRT are efficacious in decreasing the levels of biochemical markers. However, markers do not replace bone mineral density measurement. Collagen type I cross linked $\mathrm{N}$-telopeptide, collagen type I cross linked C-telopeptide, and osteocalcin are the most promising markers.

See end of article for authors' affiliations

Correspondence to: Dr Erbil Dogan, Dokuz Eylul Universitesi Tip Fakultesi, Kadin Hastaliklari ve Dogum Anabilim Dali, Inciralti/Izmir 35340 Turkey; erbil.dogan@ deu.edu.tr

Submitted 28 March 2002 Accepted 10 September 2002

\section{BONE TISSUE PHYSIOLOGY}

Bone tissue is formed by the precipitation of hydroxyapatite crystals on an organic protein matrix. Ninety nine per cent of total body calcium and phosphorus are found in bone, where they are amorphously stored on the extracellular matrix, and hydroxyapatite crystals are formed. These crystals precipitate on the collagen matrix and form the bone tissue. Glycoproteins and proteoglycans participate in binding these crystals to the matrix. Seventy per cent of bone tissue consists of minerals and the rest is organic in origin. Ninety five per cent of these minerals are in the form of calcium and phosphorus crystals. Organic bone tissue is mainly made up of a protein matrix (98\%). Only $2 \%$ is of cellular origin, but this is essential for bone metabolism. Osteoid matrix principally consists of type I collagen $(90 \%)$. Other matrix proteins are proteoglycans and non-collagenous proteins, which are osteocalcin (bone gamma-carboxyglutamic acid (GLA) protein, matrix GLA protein, osteonectin, and cellular binding proteins (fibronectin, bone sialoproteins, etc). Constituents of bone tissue are shown in fig 1.

There are two major types of bone: cortical (compact) and trabecular (spongious). Cortical bone constitutes approximately $90 \%$ of the skeleton. It is found mainly in the diaphysis of long bones and on the surfaces of flat bones. Cortical bone is composed of a concentric compact bone mass located around a central canal (haver- sian systems). There are blood vessels, lymphatics, nerves, and connective tissue in the haversian canals. Trabecular bone is found in the distal ends of long bones and in the inner surfaces of flat bones, and it involves bone marrow. Cortical bone functions as a mechanical support, while trabecular bone is involved in metabolic processes. The cellular part of bone is composed of osteoblasts, osteocytes, and osteoclasts. Osteoblasts are pleuripotent stromal cells originating from bone marrow. They are responsible for production and subsequent mineralisation of bone matrix. Osteoblasts synthesise type I collagen, growth factors, and non-collagenous proteins. In other words, these cells are responsible for bone formation. Osteoclasts are multinucleated giant cells responsible for the bone resorption. Osteocytes are flat small cells found in bone matrix, which are thought to play a part in the oestrogenic response to mechanical stimuli.

Since bone formation and resorption are continuing processes, there is a dynamic metabolism in bone tissue. Bone resorption precedes the bone formation. New bone formation (bone remodelling) occurs both in the cortical and in the trabecular bone. Mechanical stress, systemic hormones, local cytokines, growth factors, prostaglandins, prostacyclins, nitric oxide, and free radicals are the main factors controlling bone remodelling. There are various factors affecting bone resorption and formation. Interleukin (IL)-1, IL-6, IL-8, IL-1 1, tumour necrosis factors, epidermal growth factor, leukaemia inhibiting factor, macrophage colony stimulating factor, granulocyte/macrophage colony stimulating factor are the stimulators of bone resorption. Interferon- $\alpha$ and IL-4 inhibits bone resorption. Stimulators of bone formation are insulin-like growth factors, transforming growth factor- $\beta$, fibroblast growth factors, bone morphogenetic proteins, and platelet derived growth factors. Bone mass is also affected by some hormones. Growth hormone, calcitonin and progesterone increases bone mass, while triiodothyronine decreases. Parathyroid hormone is a potent stimulator of bone resorption, but it is also suggested that low levels of parathyroid hormone

Abbreviations: $B M D$, bone mineral density; $C T x$ collagen type I cross linked C-telopeptide; D-pyr, deoxypyridinoline; GLA, gamma-carboxyglutamic acid; HRT, hormone replacement therapy; IL, interleukin; NTx, collagen type I cross linked $\mathrm{N}$-telopeptide; pyr, pyridinoline 
Box 1: Potential regulatory factors in bone remodelling

Factors affecting bone resorption

Stimulators

- Interleukin (IL)-1, IL-6, IL-8, IL-1 1

- Tumour necrosis factor.

- Epidermal growth factor.

- Leukaemia inhibiting factor.

- Macrophage colony stimulating factor

- Granulocyte/macrophage colony stimulating factor. Inhibitors

- Interferon- $\gamma$.

- IL-4

Stimulators of bone formation

- Insulin-like growth factor.

- Transforming growth factor beta.

- Fibroblast growth factor.

- Bone morphogenetic proteins.

- Platelet derived growth factor.

has anabolic effect on bone tissue via transforming growth factor- $\beta$ and insulin-like growth factors. ${ }^{1}$ Factors affecting bone resorption and formation are listed in box 1 .

\section{OESTROGENS, BONE TISSUE, AND CYTOKINES}

Oestrogens are essential for the formation and maintenance of sufficient bone mass in women. Oestrogen receptors are found both on osteoblasts and on osteoclasts. Oestrogens increase osteoblastic activity by a direct effect on them. They have two different effects on vitamin D: first, they increase vitamin D receptors on osteoblasts, and secondly they activate vitamin D to increase intestinal calcium absorption. Oestrogens directly and rapidly inhibit lysosomal enzyme production in the osteoclasts and thus decrease bone resorption. Oestrogens also have indirect effects on bone metabolism by cytokines. They decrease catabolic factors like IL-1 and IL-6, tumour necrosis factor- $\alpha$, and granulocyte/macrophage colony stimulating factor; they increase anabolic factors like transforming growth factors and insulin like growth factors. ${ }^{2}{ }^{3}$ Tumour necrosis factor- $\alpha$ and IL- 1 are found to be raised in the peripheral blood monocytes of ovariectomised patients. ${ }^{4}$ Moreover, these two cytokines are found to be increased in the ovariectomised rats' bone marrow, which is reversed by oestrogen replacement. ${ }^{5}$ IL- 6 is a potent stimulator of bone resorption and it is known that ovariectomy increases IL-6 production in bone marrow cells. ${ }^{6}$ IL-6 is an important mediator in bone turnover. Ovariectomy in mice with an interleukin defect does not change bone turnover rate in the favour of bone loss. ${ }^{7}$

\section{CHANGES IN BONE MASS}

Peak bone mass is one of the main factors influencing both the future bone mass and the probable fracture risk. Peak bone mass is formed long before menopause and bone loss starts before that (fig 2). Bone loss is not prominent in men, but in women, a rapid loss begins with menopause. Genetic factors, nutritional status, physical activity, and gonadal status effect peak bone mass. Peak bone mass is high in AfricanAmericans, but low in Asians and Japanese. Bone formation and resorption occur under the control of systemic hormones and mechanical stimuli via the mediators like local cytokines and growth factors. Two factors affecting bone metabolism are obesity and smoking. Bone resorption is decreased in obesity and there is an inverse relation between body mass index and bone resorption $(r=-0.26 \mathrm{p}<0.01)$. Smoking is associated with a $4 \%$ lower bone mineral density (BMD) compared with non-smokers. ${ }^{8}$

Peak bone density in the femur is reached at the end of the third decade and starts to decline in the fourth decade. Spinal bone loss begins in the third decade. Vertebral bone is more susceptible to oestrogen deficiency, but until menopause bone loss is minimal. Bone turnover frequency increases in menopause, which is a consequence of oestrogen deficiency. Normally, there is a balance between bone formation and resorption, known as the remodelling balance. After the menopause, the bone formation rate is less than the resorption rate, which results in a negative remodelling balance, and it is related to aging rather than oestrogen deficiency. In oestrogen deficiency, rapid changes are seen in trabecular bone, and the weakening of cortical bone follows it. In this case, bone resorption and bone formation in trabecular bone are eight times more rapid than cortical bone. After the fourth decade, trabecular bone resorption exceeds formation by a rate of $0.7 \%$ per year. This gap increases after menopause, where $5 \%$ of trabecular bone is lost each year. This accelerated bone loss continues for 10-15 years, and 20 years after menopause, $50 \%$ of trabecular and $30 \%$ of cortical bone are lost. ${ }^{9}$

There are some very important points about postmenopausal hormone replacement therapy (HRT). First, most of the hormone regimens cannot replace the reduced bone mass totally. HRT can increase bone mass by $2 \%-7 \%$ per year, but after three years of treatment no further increase is seen, and bone mass is stabilised..$^{10}$ HRT will prevent bone loss and reduce future fracture risk, but after cessation of treatment there is a rapid bone loss in the first year. This is a reason to

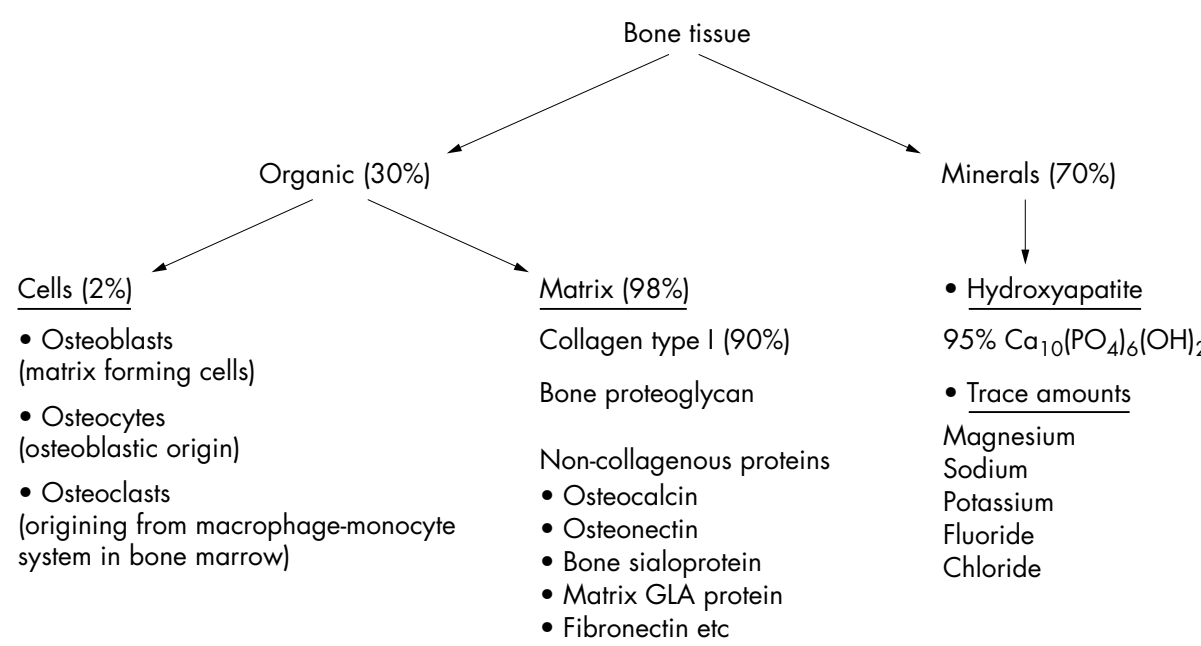

Figure 1 Constituents of bone tissue. 


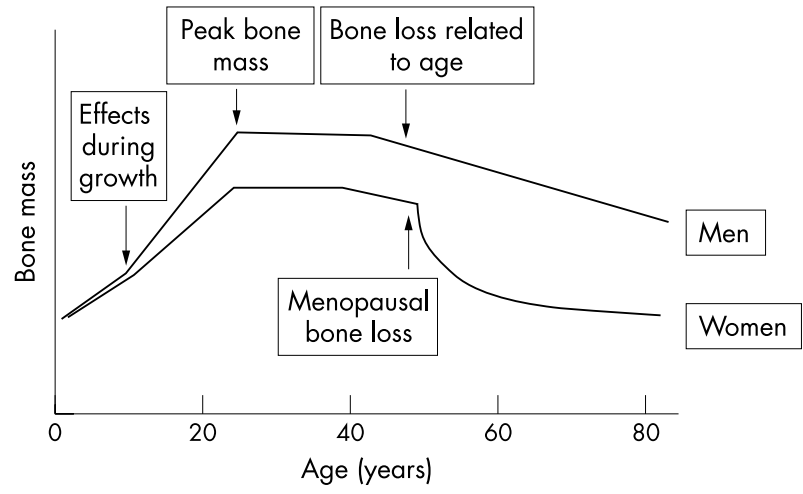

Figure 2 Age related alterations in bone mass in men and women. Note the accelerated bone loss after the menopause. Bone loss is less prominent in men compared with women.

continue therapy for long periods. According to retrospective cohort studies, BMD measurements of postmenopausal women who have used oestrogen for long periods (5-10 years) were higher than the never users. ${ }^{11}$ This means that although bone loss restarts after discontinuing HRT, the BMD level is still higher than never users. There may be a useful residual effect of oestrogen.

\section{BIOCHEMICAL MARKERS OF BONE METABOLISM}

Biochemical markers of bone metabolism are divided into two groups as bone resorption and formation markers (box 2). ${ }^{12}$ When bone turnover is increased both resorption and formation markers are raised. By this way, these markers can be used in the assessment of bone turnover rate. Bone turnover rate in postmenopausal women is higher than the perimenopausal women, which is detected by an increase in bone resorption markers followed by an increase in bone formation markers. This increased bone turnover can be diminished by HRT and this can be demonstrated by a decrease in bone formation and resorption markers. ${ }^{13}$

\section{(A) Bone formation markers}

The oldest bone formation marker is total alkaline phosphatase. It is thought to participate in the initiation of bone mineralisation. Changes in total alkaline phosphatase activity are useful as a therapeutic marker in Paget's disease, rickets, osteomalacia, renal osteodystrophy, and for monitoring the healing of new fractures, but are less useful in osteoporosis and other disorders of bone. It has a low sensitivity and specificity. Total alkaline phosphatase has two isoenzymes: bone and hepatic. Bone specific alkaline phosphatase measurements can be used as a bone formation marker in order to

\section{Box 2: Biochemical markers of bone metabolism}

\section{Bone formation markers}

- Serum osteocalcin.

- Serum total and bone specific alkaline phosphatase.

- Serum procollagen type I carboxy terminal propeptide.

- Serum procollagen type I amino terminal propeptide.

Bone resorption markers

- Urinary hydroxyproline.

- Urinary galactosyl hydroxylysine.

- Tartrate resistant acid phosphatase.

- Urinary total and free pyridinoline.

- Urinary total and free deoxypyridinoline

- Urinary collagen type I cross linked N-telopeptide.

- Urinary collagen type I cross linked C-telopeptide.

- Serum type I collagen carboxy terminal telopeptide. improve sensitivity and specificity. Postmenopausal women have higher levels of bone specific alkaline phosphatase than premenopausal women have.

Serum osteocalcin, also known as bone GLA protein, is another bone formation marker. It is a bone specific non-collagenous protein. Osteocalcin is synthesised by osteoblasts, and deposited mainly in the extracellular matrix of bone, but a small amount enters blood. Serum osteocalcin is a sensitive and specific marker of osteoblastic activity and its serum levels reflect the rate of bone formation. It is thought to be synthesised and secreted during deposition and maintenance of mineral rather than matrix synthesis. Osteocalcin is filtered by the kidneys, so serum levels are dependent on renal function.

Extension peptides of procollagen type I are other bone formation markers, which are removed by specific proteases from procollagen type I during the process of conversion to collagen. These peptides have amino and carboxy terminals, which are named accordingly as either procollagen type I carboxy terminal propeptide or procollagen type I amino terminal propeptide. When the conversion of procollagen to collagen is increased, these peptides are released into the circulation, so their serum levels may be used as a bone formation marker. Serum concentrations are increased in disorders characterised by high bone turnover such as hyperparathyroidism, hyperthyroidism, osteomalacia, Paget's disease, and metastatic bone cancer. There is a slight but insignificant increase in procollagen type I carboxy terminal propeptide after the menopause..$^{14}$ These two peptides are less valuable bone formation markers than osteocalcin.

\section{(B) Bone resorption markers}

Urinary calcium measurement is the cheapest bone resorption marker, but its sensitivity is low. Urinary excretion of calcium depends upon the renal threshold for calcium and dietary calcium intake. Another marker is urinary hydroxyproline, which is an amino acid in the collagen structure. Urinary hydroxyproline excretion will increase when collagen degradation is accelerated during bone resorption. However, the majority of the hydroxyproline derived from the breakdown of collagen is reabsorbed by the renal tubules and degraded in the liver. Only $10 \%$ of hydroxyproline is excreted in the urine. Ingestion of collagen rich foods such as meat may also increase the level of urinary hydroxyproline. As a result, hydroxyproline is not an ideal marker of bone resorption, but when measured correctly it can provide useful information. Hydroxylysine is another amino acid in the collagen structure. It has two glycoside forms, which are detected in urine. Galactosyl-hydroxylysine appears to be specific for bone collagen degradation. It is neither affected by diet nor reused by the body, so it seems to be a more sensitive marker compared with urinary hydroxyproline.

Acid phosphatase is a lysosomal enzyme found in bone, cartilage, prostate, thrombocytes, erythrocytes, and spleen. Although it has many isoenzymes, tartrate resistant isoenzyme is the one specific to bone, so measurement of tartrate resistant acid phosphatase level may give us an idea about bone resorption. However, its sensitivity is debated, compared with urinary hydroxyproline. ${ }^{15}$

Pyridinoline (pyr) and deoxypyridinoline (D-pyr) are the most promising markers of bone resorption. Type I collagen structure is shown in fig 3. Peptide chain of collagen has amino (N) and carboxy (C) terminals from which $\mathrm{N}$-telopeptides and C-telopeptides are covalently bound to collagen by pyr and D-pyr cross links. When collagen is degraded, pyr and D-pyr are released into the circulation, where $20 \%$ is free and the remaining is protein bound. The protein bound fraction binds either to $\mathrm{N}$-telopeptide, as collagen type I cross linked N-telopeptide (NTx) or to C-telopeptide, as collagen type I cross linked C-telopeptide 


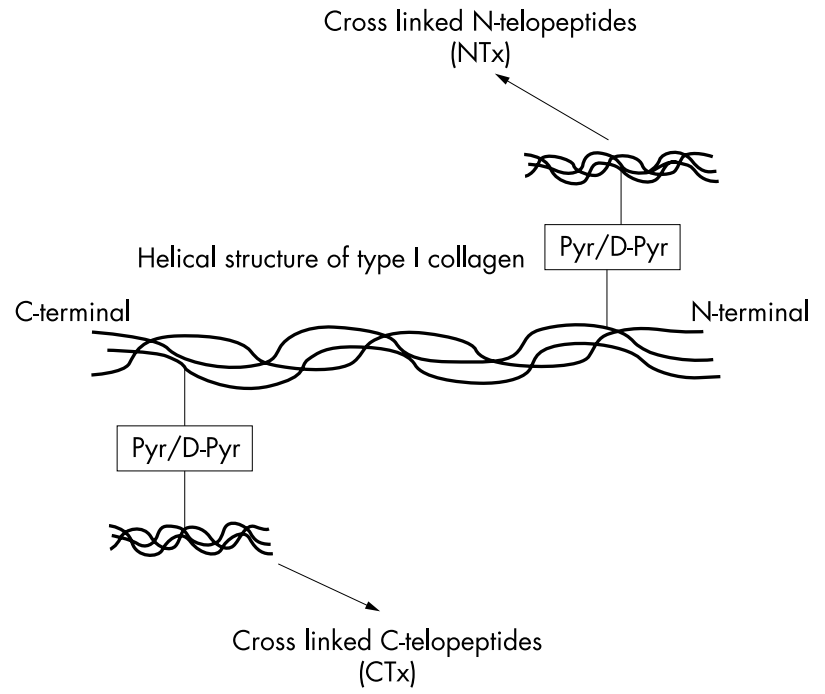

Figure 3 Cross linked $\mathrm{N}$ - and C-telopeptides of type I collagen. The pyridinoline cross links occur essentially at two intermolecular sites in the collagen fibril: aminotelopeptides are linked to a helical site at residue 930 (NTx), and carboxytelopeptides are linked to helical residue 87 (CTx).

(CTx). Protein bound fraction of pyr and D-pyr in the circulation is subjected to renal degradation resulting with $40 \%$ free and $60 \%$ protein bound forms in urine. As a conclusion, total, free, or protein bound (NTx, CTx) pyr and D-pyr can be measured as bone resorption markers. Urinary pyr and D-pyr levels increase $50 \%-100 \%$ with menopause and return to premenopausal levels with HRT. ${ }^{16}$

During HRT, there is a decrease in bone resorption markers followed by a decline in bone formation markers. Maximum effect may be seen after 3-6 months of treatment. Samples for marker measurements should be taken in the morning time after an overnight fasting, since satiety and circadian changes may affect the results.

\section{RELATION OF HRT, BIOCHEMICAL MARKERS OF BONE, AND BMD}

BMD measurement is regarded as the best approach for screening individuals with risk of osteoporosis. BMD is also the major determinant of fracture risk. Other factors affecting fracture risk are the bone turnover rate and the microarchitectural structure of the bone. However, BMD cannot assess the actual bone turnover. Estimation of bone formation and bone resorption by biochemical markers may be particularly valuable in identifying individuals who lose rapidly their bone mass like postmenopausal women. Bone resorption markers are therefore important in the early identification of patients who require HRT or other antiresorptive therapies.

The potential use of bone markers in clinical practice may be categorised as follows: (1) monitoring effectiveness of therapy; (2) monitoring patient's therapy compliance; (3) prediction of bone loss and fracture risk; (4) prediction of bone mass; and (5) selection of patients for antiresorptive therapy.

Both urine and serum CTx have shown decreases of $70 \%$ or more from baseline after bisphosphonate therapies, and approximately $50 \%$ in hormone replacement therapies. The maximum effects may be seen after 3-6 months of treatment. In a prospective randomised study with 153 postmenopausal women taking HRT, Bjarnason and Christiansen measured urinary CTx and BMD levels after three years of follow up. ${ }^{17}$ Maximum decrease in CTx levels occurred at six months, which was significantly correlated to three year bone mass response. Ravn et al used alendronate $5 \mathrm{mg}$ /day in 1202 postmenopausal women, and found that decrease in NTx $(r=$
$-0.31)$ and osteocalcin $(r=-0.25)$ levels at six months was the predictor of BMD increase at two years. ${ }^{18}$ Riis et al followed up 182 postmenopausal women for 15 years and found that baseline bone mass and rate of loss predisposed to the same extent to fractures by a ratio of two. ${ }^{19}$ However, if both low bone mass and rate of loss were present, the ratio increased to about three. Therefore, identification of fast bone losers at menopause is as important as BMD measurement for the prediction of future fracture risk.

Lindsay et al investigated 428 postmenopausal osteoporotic women in a randomised controlled study giving HRT plus alendronate ( $10 \mathrm{mg} /$ day) to one group and HRT plus placebo to the other group..$^{20}$ Serum bone specific alkaline phosphatase and urinary NTx levels were measured for one year. There was significant decrease in both markers at six months and one year of treatment in the HRT plus alendronate group compared with the controls. Similarly, BMD level at one year was higher in the HRT plus alendronate group. In a prospective randomised study, Trraş et al showed that alendronate treatment alone or in combination with HRT decreased bone turnover and increased spinal BMD at the end of one year more effectively than HRT alone. ${ }^{21}$

Alendronate treatment can normalise bone turnover and increase BMD in osteoporotic women. Kyd et al used alendronate $(10 \mathrm{mg} /$ day $)$ for one year in 30 postmenopausal women. ${ }^{22}$ After three months, urinary NTx decreased $50 \%$ and at the end of one year the mean per cent increases in BMD at the lumbar spine and femoral neck were $+4.6 \%$ and $+3.5 \%$, respectively. In a randomised, double blind, multicentre study (the PEPI trial) (23 $^{23}$ with 239 postmenopausal women, BMD and bone turnover markers (CTx, NTx, pyr, osteocalcin, bone specific alkaline phosphatase) were measured for three years of follow up. ${ }^{23}$ Maximum decrease in markers was detected at the end of the first year in the HRT group. BMD increases were $3.23 \%$ and $5.40 \%$ at first and third years, respectively. In this study, the authors concluded that markers do not seem to have a significant predictive value for BMD increase. BMD changes over one year can be best predicted by a combination of variables including baseline age, body mass index, and BMD, plus both the baseline and per cent changes in NTx and bone specific alkaline phosphatase levels. The PEPI trial is one of the rare studies concluding that bone markers offer little information for predicting BMD changes for postmenopausal women.

Both oral ${ }^{24}$ and transdermal ${ }^{25}$ HRT are equally effective in slowing down the bone turnover. Therefore, HRT route is not important. Basal marker levels may be useful in determining the subsequent BMD increase. BMD increase at the end of one year after HRT was greater in patients with high basal urinary NTx and serum osteocalcin levels. ${ }^{24}{ }^{26}$ Tibolon also slows down bone turnover and increases BMD. ${ }^{27}$

Our group reported on 66 women with natural menopause who were prospectively investigated ${ }^{28}$ Twenty three patients received oral and 22 patients received transdermal oestrogen and progesterone. Alendronate was added to oral HRT in the third group of 21 patients with a BMD less than $100 \mathrm{mg} / \mathrm{cm}^{3}$. Urinary NTx levels decreased significantly in all groups after treatment compared with the basal levels. However, the decreases in NTx levels were not statistically significant between the groups. In other words, addition of alendronate to HRT does not result with an additional decrease in urinary NTx level $(p>0.05)$.

In conclusion, biochemical markers of bone reflect acute changes in bone turnover rate. Bone turnover decreases in postmenopausal women receiving HRT, and this may be demonstrated by the decrease in the marker levels. Changes in marker levels may be used to monitor the effectiveness of therapy. Decrease in marker levels with HRT may be the early predictor of subsequent BMD increase, and possible reduction in fracture risk. However, marker measurements cannot replace BMD measurement, and no marker is ideal yet. Therefore, bone markers should be used in combination with BMD 
to monitor the therapy. NTx, CTx, and osteocalcin are the most promising markers.

\section{Authors' affiliations}

Erbil Dogan, Cemal Posaci, Dokuz Eylul University Faculty of Medicine, Department of Obstetrics and Gynaecology, Izmir, Turkey

\section{REFERENCES}

1 Kurland ES, Cosman F, McMahon DJ, et al. Parathyroid hormone as a therapy for idiopathic osteoporosis in men: effects on bone mineral density and bone markers. J Clin Endocrinol Metab 2000;85:3069-76.

2 Pacifici $R$, Brown C, Puscheck E, et al. Effect of surgical menopause and estrogen replacement on cytokine release from human blood mononuclear cells. Proc Natl Acad Sci U S A 1991;88:5134-8.

3 Posaci C, Altunyurt S, Islekel $\mathrm{H}$, et al. Effects of HRT on serum levels of IGF-I in postmenopausal women. Maturitas 2001;40(1):69-74.

4 Pacifici R, Rifas L, McCracken R, et al. Ovarian steroid treatment blocks a postmenopausal increase in blood monocyte interleukin 1 release. Proc Natl Acad Sci U S A 1989;86:2398-402.

5 Kimble RB, Vannice JL, Bloedow DC, et al. Interleukin- 1 receptor antagonist decreases bone loss and bone resorption in ovariectomized rats. J Clin Invest 1994:93:1959-67.

6 Girasole G, Jilka RL, Passeri G, et al. 17 beta-estradiol inhibits interleukin- 6 production by bone marrow-derived stromal cells and osteoblasts in vitro: a potential mechanism for the antiosteoporotic effect of estrogens. J Clin Invest 1992;89:883-91.

7 Poli V, Balena R, Fattori E, et al. Interleukin-6 deficient mice are protected from bone loss caused by estrogen depletion. EMBO 1994;13:1 189-96.

8 Lindsay R. Prevention and treatment of osteoporosis. Lancet 1993;341:801-5

9 Kanis JA. Are oestrogen deficiency and hormone replacement a distraction to the field of osteoporosis? Osteoporos Int 1998;8(suppl 1):51-6.

10 Delmas PD. HRT in the prevention and treatment of osteoporosis. J Epidemiol Biostat 1999:4:155-63.

11 Garnero P, Delmas PD. Biochemical markers of bone turnover: clinical usefulness in osteoporosis. Ann Biol Clin 1999;57:137-48

12 Delmas PD. Biochemical markers of bone turnover for the clinical investigation of osteoporosis. Osteoporos Int 1993:3/suppl 11:81-6.

13 Brincat M, Galea R, Baron YM, et al. Changes in bone collagen markers and in bone density in hormone treated and untreated postmenopausal women. Maturitas 1997;27(2): 171-7.

14 Kraenzlin ME, Lau KH, Liang L, et al. Development of an immunoassay for human serum osteoclastic tartrate-resistant acid phosphatase. J Clin Endocrinol Metab 1990;71:442-51.
15 Uebelhart D, Schlemmer A, Johansen JS, et al. Effect of menopause and hormone replacement therapy on the urinary excretion of pyridinium cross-links. J Clin Endocrinol Metab 1991;72:367-73.

16 Bjarnason NH, Christiansen C. The influence of thinness and smoking on bone loss and response to hormone replacement therapy in early postmenopausal women. J Clin Endocrinol Metab 2000:85:590-6.

17 Bjarnason NH, Christiansen C. Early response in biochemical markers predicts long-term response in bone mass during hormone replacement therapy in early postmenopausal women. Bone 2000;26:561-9.

18 Ravn P, Hosking D, Thompson D, et al. Monitoring of alendronate treatment and prediction of effect on bone mass by biochemical markers in the early postmenopausal intervention cohort study. J Clin Endocrinol Metab 1999;84:2363-8.

19 Riis BJ, Hansen MA, Jensen AM, et al. Low bone mass and fast rate of bone loss at menopause: equal risk factors for future fracture: a 15 -year follow-up study. Bone 1996;19:9-12.

20 Lindsay R, Cosman F, Lobo RA, et al. Addition of alendronate to ongoing hormone replacement therapy in the treatment of osteoporosis: a randomized, controlled clinical trial. J Clin Endocrinol Metab 1999:84:3076-81

21 Tıraş MB, Noyan V, Yildiz A, et al. Effects of alendronate and hormone replacement therapy, alone or in combination, on bone mass in postmenopausal women with osteoporosis: a prospective, randomized study. Hum Reprod 2000;15:2087-92.

22 Kyd PA, De Vooght K, Kerkhoff F, et al. Clinical usefulness of biochemical resorption markers in osteoporosis. Ann Clin Biochem 1999;36:483-91

23 Marcus R, Holloway L, Wells B, et al. The relationship of biochemical markers of bone turnover to bone density changes in postmenopausal women: results from the Postmenopausal Estrogen/Progestin Interventions (PEPI) trial. J Bone Miner Res 1999;14:1583-95.

24 Rosen CJ, Chesnut CH 3rd, Mallinak NJ. The predictive value of biochemical markers of bone turnover for bone mineral density in early postmenopausal women treated with hormone replacement or calcium supplementation. J Clin Endocrinol Metab 1997;82:1904-10.

25 Peris P, Alvarez L, Monegal A, et al. Biochemical markers of bone turnover after surgical menopause and hormone replacement therapy. Bone 1999;25:349-53

26 Masahashi T, Negoro Y, Kuroki T, et al. Biochemical markers of bone turnover during hormone replacement therapy. Int J Gynaecol Obstet 1999;64:163-6.

27 Bjarnason NH, Bjarnason K, Haarbo J, et al. Tibolone: prevention of bone loss in late postmenopausal women. J Clin Endocrinol Metab 1996:81:2419-22.

28 Posaci C, Altunyurt S, Islekel $\mathrm{H}$, et al. The values of urinary NTx in postmenopausal women undergoing hormone replacement therapy; the role of additional alendronate therapy. Maturitas 2002:42:281-6. 